\title{
Obituário de Osvaldo Rodrigues da Cunha (1928-2011)
}

\author{
Marinus Steven Hoogmoed \\ Coordenação de Zoologia, Museu Paraense Emílio Goeldi \\ Teresa Cristina Sauer de Avila-Pires \\ Coordenação de Zoologia, Museu Paraense Emílio Goeldi \\ Nelson Sanjad \\ Coordenação de Comunicação e Extensão, Museu Paraense Emílio Goeldi
}

No dia 11 de março de 2011, faleceu Osvaldo Rodrigues da Cunha (Figuras 1, 2 e 7), pesquisador aposentado do Museu Paraense Emílio Goeldi (MPEG), fundador do setor de herpetologia e da coleção herpetológica da instituição, incansável estudioso dos répteis da Amazônia e também da história científica da região, especialmente com relação ao MPEG e às pessoas a ele vinculadas ao longo do tempo. Cunha faleceu em Belém, aos 82 anos de idade, depois de três anos com a saúde debilitada e de ter perdido a visão.

Osvaldo Cunha nasceu no dia 6 de abril de 1928, em Belém do Pará, Brasil, como primogênito do casal português Carlos Pereira da Cunha e Ana Cerqueira Rodrigues da Cunha, os quais tiveram ainda mais um filho e duas filhas. Cursou a escola primária e o ginásio em Belém, até 1946. Segundo seu próprio testemunho, recusou-se a seguir o curso universitário oferecido à sua época, pois o considerou inadequado aos estudos de história natural a que queria se dedicar, influenciado por sua convivência, desde menino, com o Parque Zoobotânico do MPEG, do qual era vizinho. Preferiu preparar-se a partir da prática do trabalho no museu e estudando nas bibliotecas dessa instituição e na do Museu Nacional do Rio de Janeiro (MNRJ), onde encontrou um vasto acervo sobre a literatura zoológica que lhe interessava. Aos poucos, foi ele mesmo adquirindo também uma valiosa biblioteca.

Em 1945, ingressou no MPEG como estagiário da então Seção de Zoologia. Iniciou estudando borboletas e besouros dos arredores de Belém, especialmente das matas de Utinga e Icoaraci, atualmente bastante reduzidas. Entre 1947 e 1954, fez algumas viagens de campo para coletar material para as coleções do MPEG no entorno da capital e no baixo Amazonas, rio Tapajós (área de Fordlândia e ao sul de Itaituba) e rio Tocantins. Em 1948, realizou, por seis meses, o serviço militar na base da Aeronáutica em Belém, período em que precisou interromper seus estudos zoológicos.

Osvaldo tinha talentos cientíicos e também artísticos. Entre 1948 e 1963, fez vários desenhos, tanto em nanquim como a lápis, retratando pessoas, paisagens e alguns animais. Reproduzimos aqui dois desses desenhos: o retrato de Emílio Goeldi (1859-1917), feito a partir de uma fotografia existente no arquivo do MPEG (Figura 3); e um hemíptero (Figura 4).

De setembro de 1949 até janeiro de 1951, Cunha estagiou na Divisão de Zoologia do MNRJ. Durante esse tempo, frequentou cursos ali ministrados em Biologia, Anatomia Comparada, Estatística aplicada à Biologia, História Natural Prática e Biologia Geral. Retornando a Belém e ao MPEG, passou a ocupar-se mais com os vertebrados, especialmente com os répteis. Cunha justificou seu novo interesse em razão da influência que recebeu de Antenor Leitão de Carvalho (1910-1985), então chefe da Seção de Répteis do MNRJ, que conhecera durante o estágio.

No dia 17 de dezembro de 1953, Osvaldo Cunha foi nomeado 'Naturalista Padrão R' do MPEG. No final de 1955, foi transferido para a Divisão de Geologia da instituição, onde trabalhou sob a orientação de Cândido Simões Ferreira, em projeto sobre a Formação 


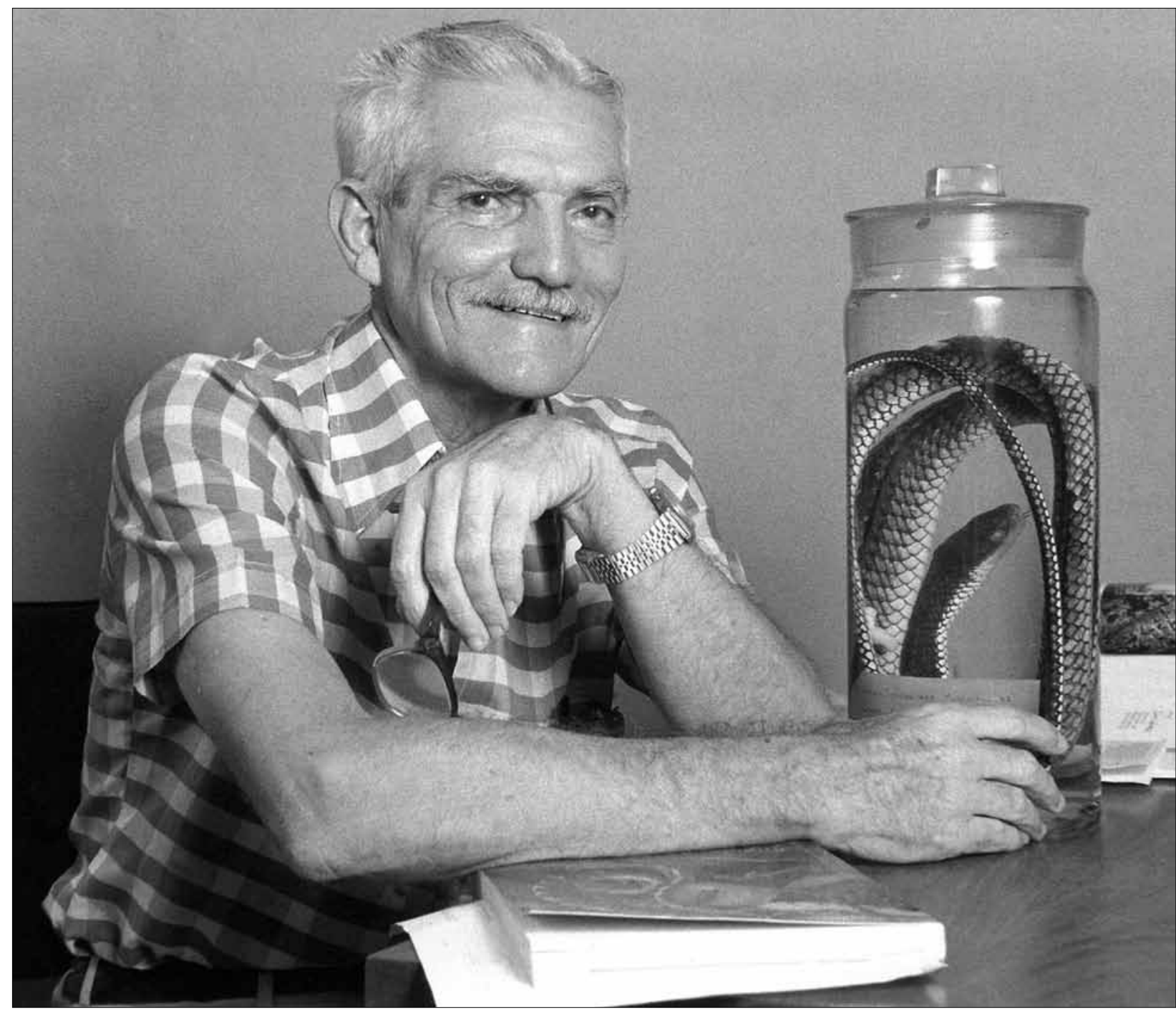

Figura 1. Osvaldo Rodrigues da Cunha em 1991, com um exemplar de Chironius carinatus (Linnaeus, 1758) da coleção herpetológica do MPEG. Fotografia de Janduari Simões. Acervo de Osvaldo Rodrigues da Cunha.

Pirabas, no leste do Pará. Entre 1957 e 1962, respondeu provisoriamente pela Divisão. As contribuições de Cunha à Geologia e Paleontologia foram reconhecidas durante o XXI Congresso Brasileiro de Paleontologia, ocorrido em Belém, em 2009. Nessa ocasião, foi homenageado por sua colaboração na reativação dos estudos e das coleções geológicas e paleontológicas do MPEG, e pela obra pioneira e detalhada sobre o conteúdo malacológico da Formação Pirabas.
Em 1962, Cunha retornou à Divisão de Zoologia do MPEG. Ainda no período em que estava na Divisão de Geologia, publicou seus primeiros trabalhos sobre os lagartos da Amazônia brasileira. A publicação de 1961, um estudo preliminar sobre os lagartos ("II. Lacertílios da Amazônia"), abordando as várias espécies então conhecidas, representou o primeiro trabalho de maior abrangência sobre um grupo da herpetofauna sulamericana. $\bigcirc$ estudo foi baseado apenas no material

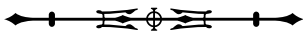




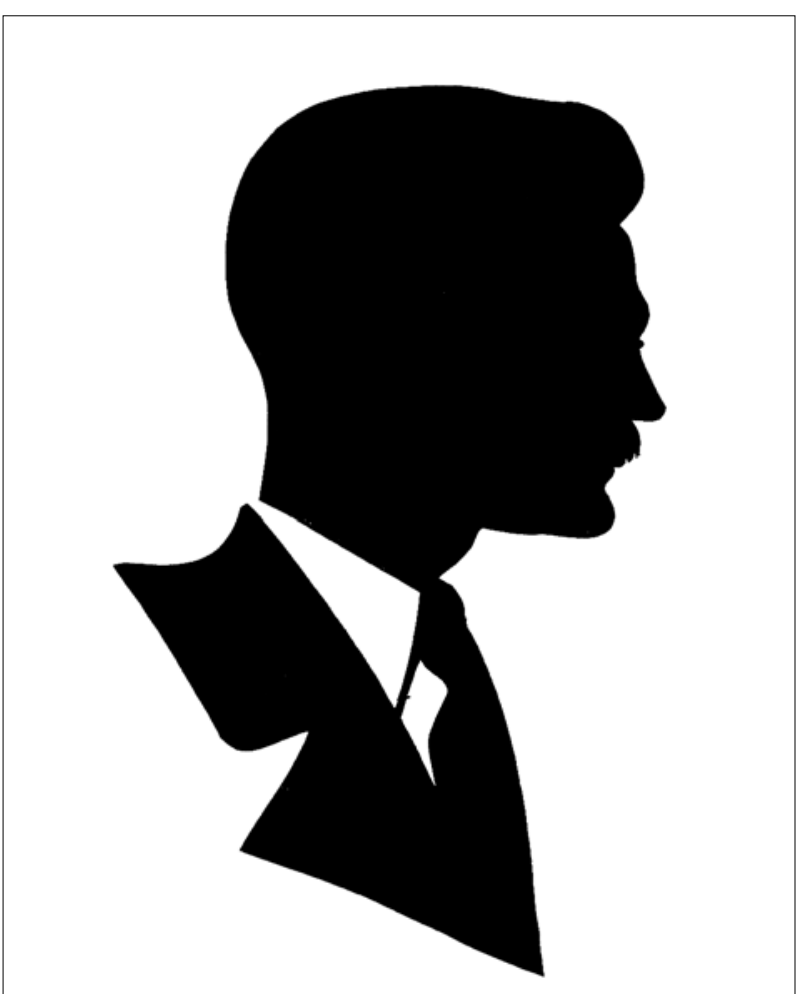

Figura 2. Silhueta de Osvaldo Rodrigues da Cunha feita por ele mesmo em 1961. Acervo de Osvaldo Rodrigues da Cunha.

da coleção do MPEG, mas esse trabalho pioneiro foi o fundamento para outros estudos mais abrangentes sobre o assunto, por exemplo, o estudo de Avila-Pires (1995, "Lizards of Brazilian Amazonia", Zoölogische Verhandelingen, n. 299), que incorporou também material dos principais museus da Europa e dos Estados Unidos. Aquele segundo trabalho de Cunha sobre lagartos foi seguido por mais seis da mesma série, até o ano de 1981. O número IV da série "Lacertílios da Amazônia" (Cunha, 1970) tratou da descrição de uma nova espécie e um novo gênero (Amapasaurus tetradactylus), baseada em dois exemplares coletados em 1960 e 1969 no então Território Federal do Amapá. Somente entre 2004 e 2006, esse lagarto veio novamente a ser coletado no Amapá. Em 2008, a espécie também foi encontrada no norte do Pará (Avila-Pires, Hoogmoed \& Rocha, 2010, Boletim do Museu Paraense Emílio Goeldi. Ciências Naturais, 5(1)).

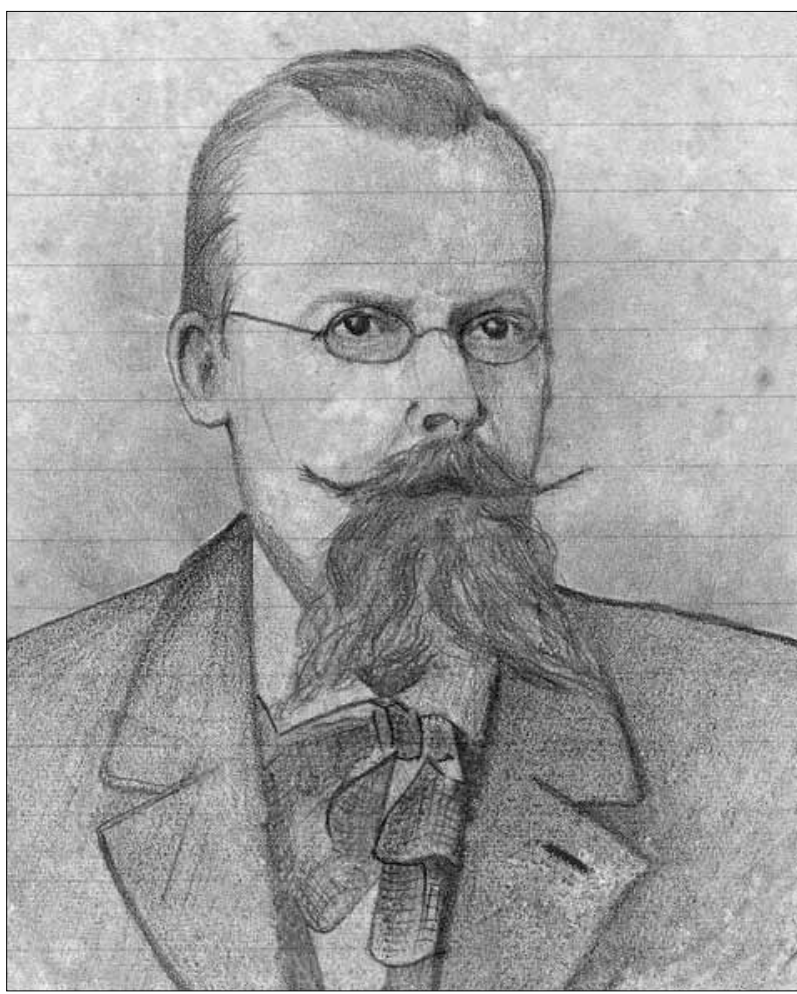

Figura 3. Desenho a lápis de Emílio Goeldi, feito por Osvaldo Rodrigues da Cunha em 1948. Acervo de Osvaldo Rodrigues da Cunha.

De setembro de 1963 até dezembro de 1964, Cunha foi estagiar com Paulo Emílio Vanzolini, diretor do Museu de Zoologia da Universidade de São Paulo (MZUSP) e herpetólogo treinado nos Estados Unidos. Esse estágio lhe permitiu aprofundar os conhecimentos sobre répteis, por ter acesso à biblioteca herpetológica de Vanzolini e por participar em trabalhos científicos, palestras, reuniões e seminários. Nesse mesmo período, encontrou também Alphonse Richard Hoge (1912-1982), chefe da Seção de Herpetologia do Instituto Butantan, em São Paulo.

Em 1966, Cunha foi nomeado 'Pesquisador em Zoologia' do MPEG. Em junho de 1965, instalou a Seção de Herpetologia da instituição, com um acervo inicial de 650 espécimes (a coleção de serpentes coletadas entre 1896 e 1916, durante as gestões de Emílio Goeldi, Jacques Huber e Emília Snethlage, havia sido enviada ao Instituto Butantan e nunca foi devolvida). No mesmo ano,

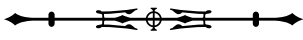




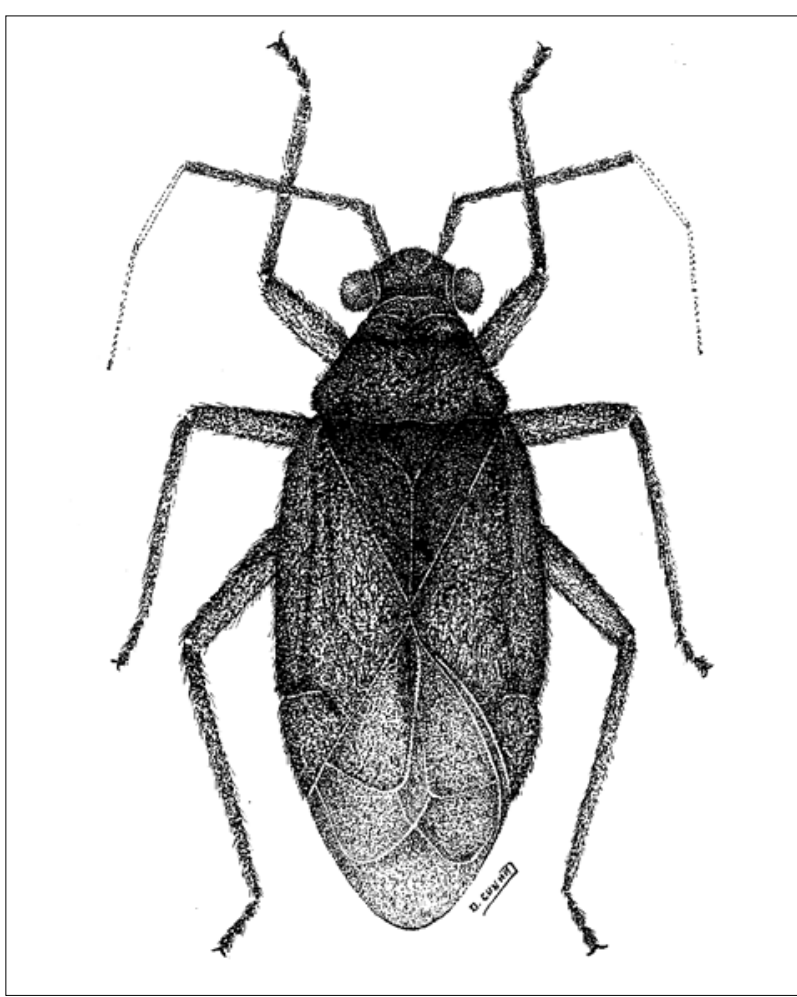

Figura 4. Desenho a nanquim de um hemíptero, feito por Osvaldo Rodrigues da Cunha no Rio de Janeiro, em 1950. Acervo de Osvaldo Rodrigues da Cunha.

Cunha começou a orientar os trabalhos herpetológicos de Francisco Paiva do Nascimento, que viria a ser seu principal colaborador, com resultados muito frutíferos para o conhecimento da herpetofauna amazônica, especialmente do Pará, e para a coleção herpetológica do MPEG. Depois da entrada de Nascimento na Seção de Herpetologia, ambos desenvolveram diversos projetos e Nascimento realizou trabalhos de campo no Amapá, Maranhão, Pará, Rondônia e Roraima, que resultaram em novas publicações por Cunha e Nascimento.

A fim de realizar um inventário o mais completo possível da herpetofauna, Cunha e Nascimento desenvolveram um sistema simples, mas efetivo, de obter exemplares para a coleção. Estabeleceram contatos com moradores em uma série de localidades no leste do Pará e, posteriormente, também no sul do estado e oeste do Maranhão (ver os mapas nos trabalhos de Cunha e Nascimento sobre os ofídios dessas áreas). Em cada um desses locais, treinaram uma pessoa para fixar os exemplares encontrados (injetando nos animais formol a $4 \%$, ou seja, uma parte de formol e nove partes de água), os quais, posteriormente, eram colocados em um tambor com álcool a 70-80\% (todo o material era acondicionado no local, já nas diluições desejadas). Aproximadamente a cada mês (ou em intervalos maiores, no caso de áreas de mais difícil acesso), eles visitavam cada um dos locais, recolhendo o material coletado. Dessa forma, exemplares encontrados no dia a dia das pessoas, inclusive durante o preparo da roça, por exemplo, puderam ser incorporados à coleção. Isso permitiu a coleta de espécies dificilmente encontradas durante expedições, por serem raras ou difíceis de detectar. Assim, a coleção herpetológica do MPEG cresceu muito em relativamente pouco tempo. Esse sistema foi especialmente útil para as serpentes, cuja coleta é usualmente esporádica, e para os répteis e anfíbios fossoriais, respectivamente, Amphisbaenidae (mãe-desaúva, cobras-de-duas-cabeças) e Gymnophiona (cobrascegas). Resultou também na coleta de uma grande série de alguns lagartos, entre os quais vale destacar Stenocercus dumerilii (Steindachner, 1867), até então conhecido apenas por uns poucos exemplares. Essa série foi a base de uma publicação com a redescrição da espécie (sob o nome Ophryoessoides tricristatus) e com notas sobre sua ecologia e distribuição (Cunha, 1981).

O abundante material obtido a partir dessas coletas constituiu a base da série pioneira e seminal intitulada "Ofídios da Amazônia", de autoria de Cunha e Nascimento, com numeração de I a XXII (o número I foi publicado por Cunha como único autor e o número XII contou com Hoge como coautor). De especial interesse foi o número X, "As cobras da região Leste do Pará" (Cunha \& Nascimento, 1978), que se tornou um clássico para estudos de serpentes da Amazônia (Figura 5). Em 1993, foi publicado um segundo livro, anunciado como segunda edição, mas que, na realidade, é mais um complemento ao primeiro livro, contendo dados 


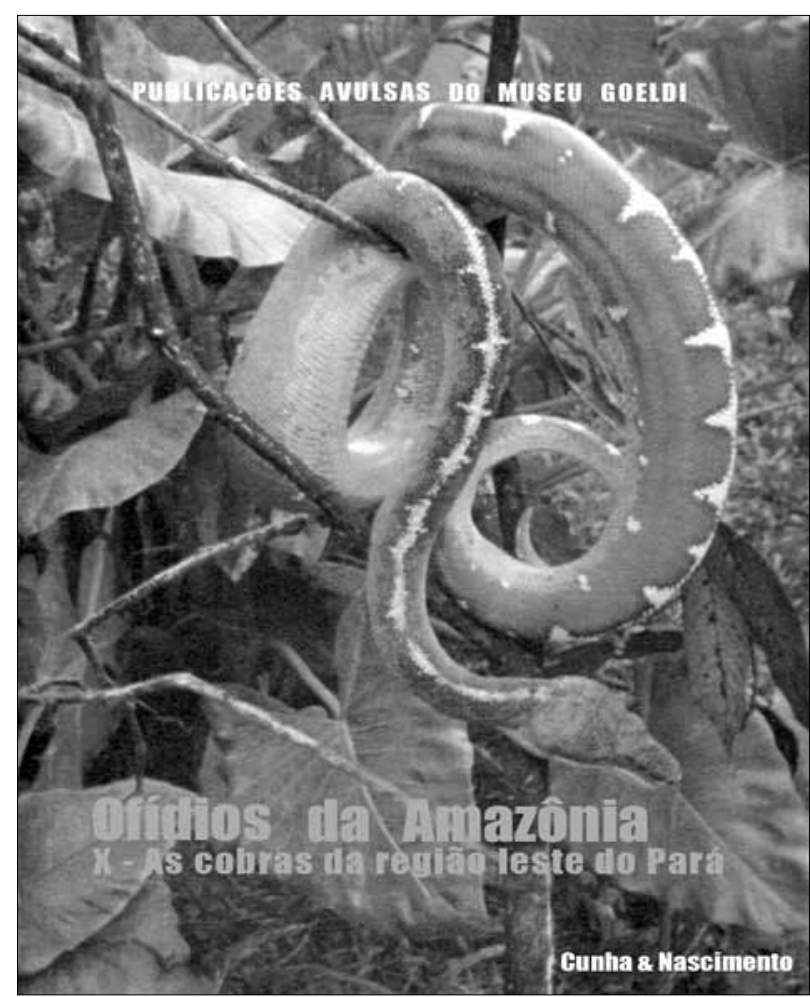

Figura 5. Capa da publicação "As cobras da região leste do Pará", de 1978.

adicionais à publicação de 1978 e outras fotos (em preto e branco), incluindo 87 espécies (12 a mais que no trabalho original). Este foi o último trabalho em herpetologia de Cunha (e também de Nascimento).

No momento da aposentadoria de Cunha, em 1990, a coleção herpetológica do MPEG contava com 38.000 exemplares, um aumento de quase 60 vezes em comparação com o número de exemplares existentes em 1965. Constituía, então, a maior coleção herpetológica com material da Amazônia brasileira e a terceira do Brasil. A coleção continuou a crescer após a aposentadoria de Cunha, contando atualmente com aproximadamente 86.000 exemplares. Ainda está entre as três maiores coleções herpetológicas do Brasil, sendo a maior com relação à herpetofauna amazônica.

Quando, em 1985, a coleção herpetológica foi transferida para um novo prédio, no Campus de Pesquisa

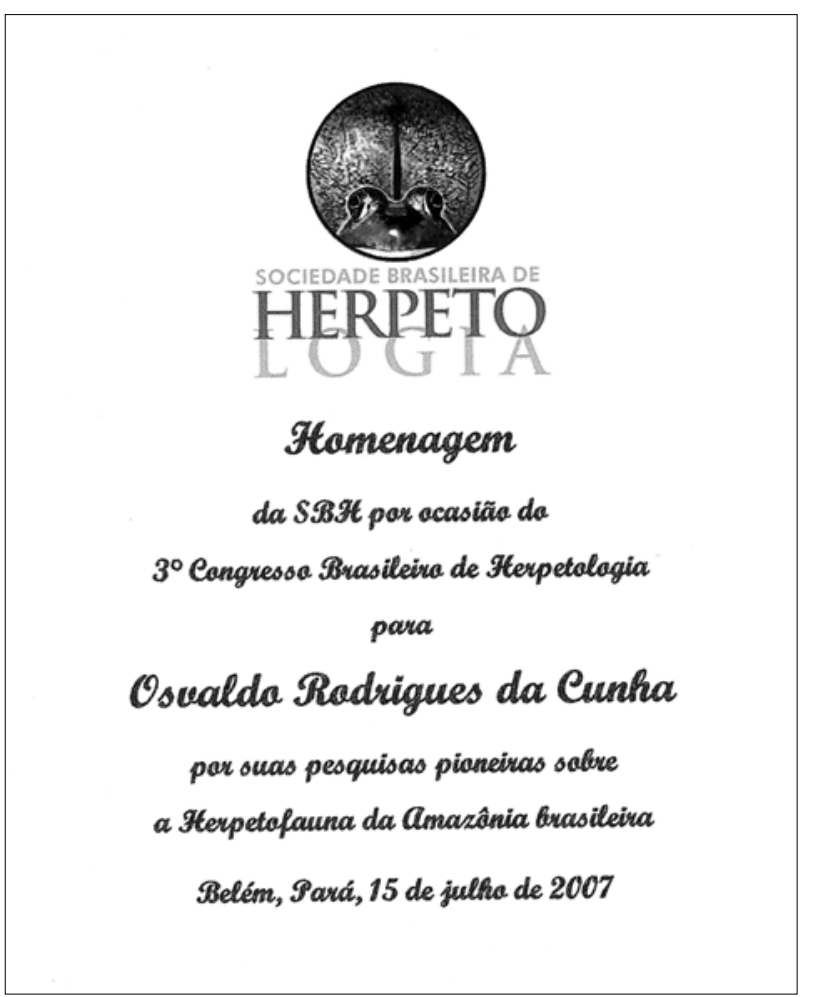

Figura 6. Homenagem da Sociedade Brasileira de Herpetologia a Osvaldo Rodrigues da Cunha, 2007.

do MPEG, Osvaldo Cunha preferiu permanecer em sua antiga sala, no Parque Zoobotânico, afastando-se da coleção. Esse foi um momento decisivo na carreira de Cunha, a partir do qual sua produção científica sobre herpetologia declinou. Depois da mudança da coleção, ele publicou apenas quatro trabalhos herpetológicos, sendo dois como terceiro coautor.

Os trabalhos sobre herpetofauna amazônica de Cunha e Nascimento formam uma boa base para estudos mais abrangentes sobre os répteis da região, os quais continuam a ser desenvolvidos por pesquisadores do MPEG e, mais recentemente, da Universidade Federal do Pará, junto com graduandos, mestrandos e doutorandos em herpetologia. Pode-se afirmar, ainda, que, ao menos em parte, Osvaldo Cunha foi o responsável pela presença desses pesquisadores nas duas instituições do Pará. Sem seu trabalho pioneiro e a coleção herpetológica que

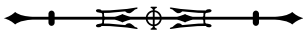




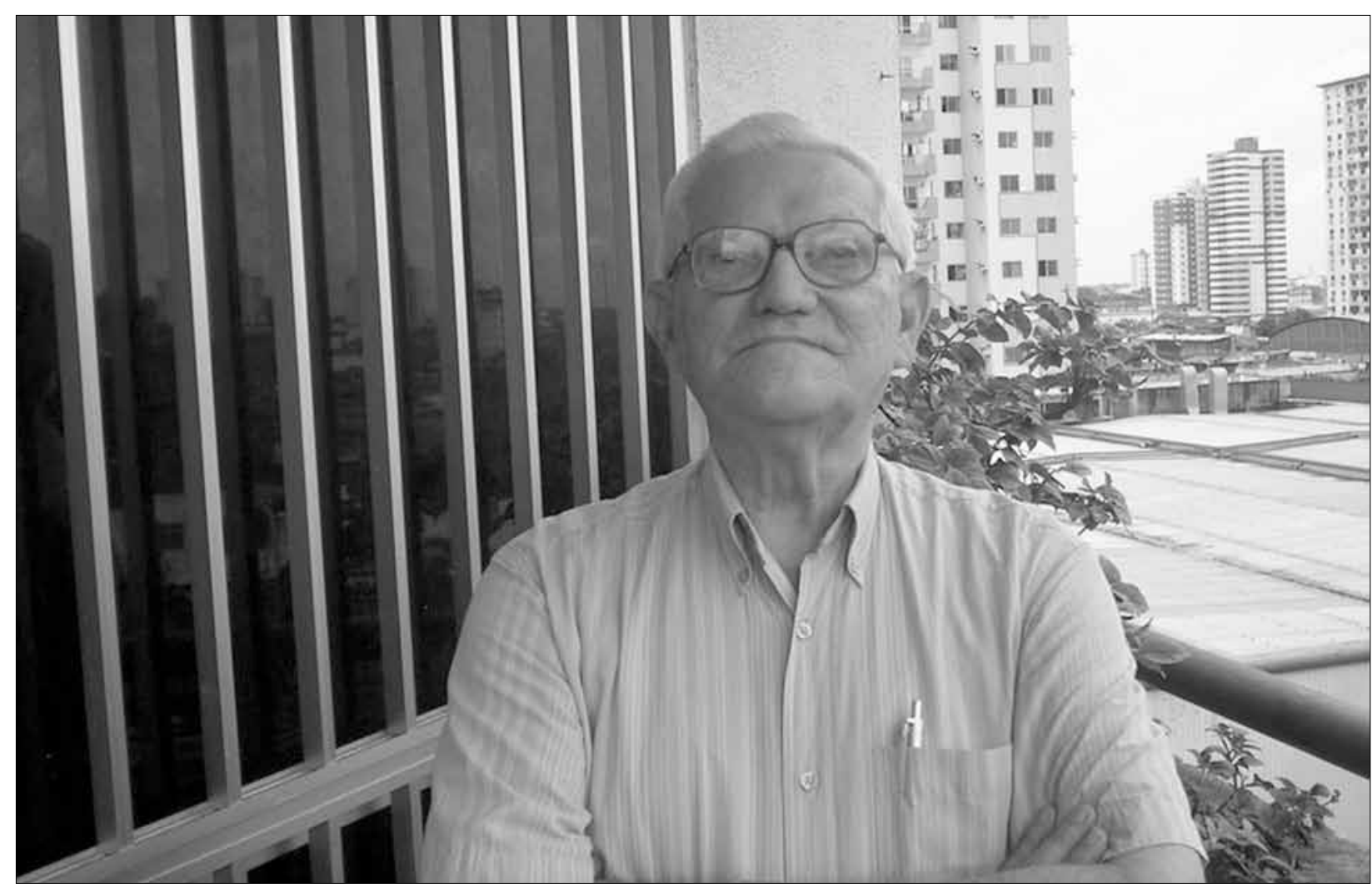

Figura 7. Osvaldo Rodrigues da Cunha em seu $80^{\circ}$ aniversário, em 2008. Fotógrafo não identificado. Acervo de Osvaldo Rodrigues da Cunha.

iniciou, o MPEG certamente não seria tão atrativo para herpetólogos de outras partes do país e do estrangeiro.

Osvaldo Cunha se aposentou aos 62 anos de idade, mas continuou trabalhando em outra área de seu interesse, a história do MPEG e dos cientistas que lá trabalharam ou por lá passaram. Um dos trabalhos mais interessantes que Cunha elaborou nesse período foi um pequeno livro (Cunha, 1991) sobre o naturalista Alexandre Rodrigues Ferreira (1756-1815), que viajou pela Amazônia entre 1783 e 1792. Nessa publicação, Cunha escreveu uma breve biografia de Rodrigues Ferreira e incluiu informações sobre outros naturalistas viajantes que estiveram na Amazônia no século XIX, além de mapas com os percursos percorridos por cada um deles. Sua intenção foi comparar o percurso das expedições e demonstrar que a viagem pioneira de Rodrigues Ferreira criou certo padrão ou estabeleceu uma referência geográfica para os que vieram depois, demarcando roteiros e pontos de coleta. Dessa forma, a publicação constitui uma fonte bastante útil sobre o trabalho dos naturalistas viajantes que coletaram os primeiros dados sobre a fauna e a flora da Amazônia.

Infelizmente, a perda da visão impediu Osvaldo Cunha de finalizar algumas biografias, deixando vários manuscritos inacabados. Em 2009, surgiu ainda uma publicação de sua autoria, sobre o botânico Jacques Huber (1867-1914), sucessor de Goeldi na direção do Museu Paraense, que havia sido anteriormente publicada, em parte, no jornal "Diário do Pará", e que foi resgatada e atualizada, com o consentimento de Cunha, por Nelson Sanjad, editor do Boletim do Museu Paraense Emílio Goeldi. Ciências Humanas. Aliás, Cunha publicou, entre 1954 e 1988, além dos artigos cientíícos, inúmeros textos em jornais de Belém, tanto sobre temas de zoologia como da história da ciência, indicando a importância que dava à divulgação do conhecimento. 
Numa primeira impressão, Cunha parecia uma pessoa fechada e difícil, mas aqueles que tiveram a oportunidade de estabelecer um contato um pouco maior com ele encontraram um homem atento, com uma memória prodigiosa e que podia conversar horas a fio sobre assuntos muito diversos, além da herpetologia. Era também uma pessoa extremamente honesta em suas opiniões e em seus trabalhos, sendo seus relatos históricos fontes fidedignas de informação. Em retrospecto, Osvaldo Cunha foi um desbravador que soube identificar áreas do conhecimento relevantes para a região, mas até então negligenciadas, dedicando-se a preencher algumas dessas lacunas. Com o falecimento de Cunha, o MPEG perdeu um grande pesquisador e o maior conhecedor da história da instituição e de seus cientistas.

No dia 11 de abril 2011, durante um tributo a Osvaldo Rodrigues da Cunha, a coleção herpetológica do Museu Paraense Emílio Goeldi foi renomeada "Coleção Herpetológica Osvaldo Rodrigues da Cunha" e uma placa com o novo nome colocada na coleção.

Este obituário foi baseado, primordialmente, na biografia de Cunha publicada em 1991, por Francisco Paiva do Nascimento, no Boletim do Museu Paraense Emílio Goeldi, série Zoologia, 7(1), número dedicado ao pesquisador, assim como em nossa convivência e nas longas conversas que tivemos com ele ao longo dos anos. Um de nós (TCSAP) chegou ao MPEG em 1983, como mestre em Zoologia, mas sem qualquer experiência com a herpetofauna amazônica. Passou, então, a trabalhar com Osvaldo Cunha e Francisco Nascimento, parceria que resultou em três publicações conjuntas e, mais do que isso, em aprendizado, amizade e admiração. $O$ primeiro autor (MSH) manteve, a partir dos anos 1960, correspondência regular com Osvaldo Cunha sobre a herpetofauna das Guianas e, em 1976, teve a oportunidade de conhecê-lo pessoalmente durante uma visita de uma semana às coleções do MPEG. Posteriormente, quando passou a realizar trabalhos de campo no Brasil com maior frequência e finalmente mudou-se para cá, o contato se estreitou. Por sua vez, NS conheceu Cunha em meados da década de 1990, quando ingressou no MPEG, tomando sua obra historiográfica como ponto de partida e inspiração para ampliar os estudos sobre a história da ciência na Amazônia, particularmente o período de fundação e reformulação do Museu Paraense, entre 1866 e 1921.

A seguir, apresentamos os taxa descritos por Osvaldo Rodrigues da Cunha, as homenagens que recebeu e a obra completa. Esta foi copiada de Nascimento (1991 [vide referência anterior]), com algumas correções e com a adição das publicações surgidas após 1990.

\section{TAXA DESCRITOS POR OSVALDO RODRIGUES DA CUNHA (ETAL.)}

(* indica novo gênero)

\section{REPTILIA, SERPENTES:}

Atractus albuquerquei Cunha \& Nascimento, 1983

Atractus alphonsehogei Cunha \& Nascimento, 1983

Atractus flammigerus snethlagae Cunha \& Nascimento, 1983

[= Atractus snethlagae Cunha \& Nascimento, 1983] Liophis carajasensis Cunha, Nascimento \& Avila-Pires, 1985 Mastigodryas bifossatus lacerdai Cunha \& Nascimento, 1978

[= Mastigodryas b. bifossatus (Raddi, 1820)] Micrurus psiches [sic!] paraensis Cunha \& Nascimento, 1973

[= Micrurus paraensis Cunha \& Nascimento, 1973] Oxyrhopus melanogenys orientalis Cunha \& Nascimento, 1993 Sibynomorphus mikani [sic] septentrionalis Cunha, Nascimento \& Hoge, 1980 [ = Sibynomorphus mikanii (Schlegel, 1837)]

\section{REPTILIA, LAGARTOS:}

*Amapasaurus tetradactylus Cunha, 1970

Arthrosaura amapaense Cunha, 1967 [= Arthrosaura reticulata (O'Shaugnessy, 1881)]

Colobosaura landii Cunha, 1977 [= Colobosaura modesta

(Reinhardt \& Lütken, 1862)]

*Colobosauroides cearensis Cunha, Lima-Verde \& Lima, 1991

Gonatodes eladioi Nascimento, Cunha \& Avila-Pires, 1987

Placosoma cipoensis Cunha, 1966 
REPTILIA, QUELÔNIO:

Kinosternon scorpioides carajasensis Cunha, 1970 [= Kinosternon s. scorpioides (Linnaeus, 1766)]

\section{INVERTEBRADOS FÓSSEIS:}

\section{Mollusca, Gastropoda}

Astrea brasiliensis Ferreira \& Cunha, 1957

Cypraea macrovoluta Ferreira \& Cunha, 1957

Diodora fortalezensis Ferreira \& Cunha, 1957

Diodora siqueirai Ferreira \& Cunha, 1957

Simpulum carlotae Ferreira \& Cunha [= Cymatium (Septra)

carlotae (Ferreira \& Cunha, 1957)]

Strombus goeldii Ferreira \& Cunha, 1957

Vasum kraatzi Ferreira \& Cunha, 1957

Xancus amazonianum Ferreira \& Cunha, 1957 [= Turbinella amazoniana (Ferreira \& Cunha, 1957)]

Xancus mauryae Ferreira \& Cunha, 1957 [(=Turbinella mauryae (Ferreira \& Cunha, 1957)]

Mollusca, Bivalvia

Atrina pirabensis Ferreira \& Cunha, 1959

Mytilus vidali Ferreira \& Cunha, 1959 [= Mytilus canoasensis vidali Ferreira \& Cunha, 1959]

Modiolus domingosi Ferreira \& Cunha, 1959

Divaricella castelensis Ferreira \& Cunha, 1959

\section{ESPÉCIES DE RÉPTEIS E ANFÍBIOS NOMEADAS EM HOMENAGEM A OSVALDO RODRIGUES DA CUNHA}

Amphisbaena cunhai Hoogmoed \& Avila-Pires, 1991

Leposoma osvaldoi Avila-Pires, 1995

Typhlonectes cunhai Cascon, Lima-Verde \& Marques, 1991 [ = Typhlonectes compressicauda (Duméril \& Bibron, 1841)]

\section{HONRARIAS RECEBIDAS}

1978 - Diploma de Honra ao Mérito, Instituto Nacional de Pesquisas da Amazônia (INPA).
1986 - Diploma e Medalha de Mérito em Zoologia, Sociedade Brasileira de Zoologia, durante o XIII Congresso Brasileiro de Zoologia, Cuiabá, Mato Grosso.

1986 - Diploma de Pesquisador Emérito do MPEG.

1991 - Grau de Comendador da Ordem do Mérito Grão Pará.

2007 - Homenagem prestada pela Sociedade Brasileira de Herpetologia durante o III Congresso Brasileiro de Herpetologia, Belém, Pará (Figura 6).

2009 - Homenagem prestada pela Sociedade Brasileira de Paleontologia durante o XXI Congresso Brasileiro de Paleontologia, Belém, Pará.

2011 - Homenagem póstuma renomeando a coleção herpetológica do Museu Paraense Emílio Goeldi como "Coleção Herpetológica Osvaldo Rodrigues da Cunha".

\section{DEDICATÓRIAS EM PUBLICAÇÕES}

1979 - Augusto Meira Filho, "Landi, esse desconhecido (O naturalista)" (p. 21).

1991 - Boletim do Museu Paraense Emílio Goeldi, série Zoologia 7(1), número dedicado a Osvaldo Rodrigues da Cunha, por ocasião de sua aposentadoria.

1998 - Márcio Martins \& M. Ermelinda Oliveira, "Natural history of snakes in forests of the Manaus region, central Amazonia, Brazil", Herpetological Natural History 6: 78-150 (p. 78).

2010 - Nelson Sanjad, "A coruja de Minerva: o Museu Paraense entre o Império e a República (1866-1907)" (p. 33).

\section{PUBLICAÇÕES DE OSVALDO RODRIGUES DA CUNHA}

AYRES, M., M. M. SAMPAIO, R. M. BARROS, L. B. DIAS \& O. R. CUNHA, 1968. Estudos preliminares sobre o número cromossômico de quelônios do gênero Podocnemis. Ciência e Cultura 20: 174-175.

AYRES, M., M. M. SAMPAIO, R. M. ARROS, L. B. DIAS \& O. R. CUNHA, 1969. A karyological study of turtles from the Brazilian Amazon Region. Cytogenetics 8: 401-409.

BARROS, R. M., M. AYRES, M. M. SAMPAIO, O. R. CUNHA \& M. F. ASSIS, 1971. Karyotypes of two subspecies of turtles from the Amazon region of Brazil. Caryologia 25(4): 463-469. 
BARROS, R. M.; M. M. SAMPAIO, M. F. ASSIS, M. AYRES \& O. R. CUNHA, 1975. A karyological study of Geoemyda punctularia punctularia (Daudin, 1802) from the Amazon region of Brazil (Chelonia, Emydidae). Acta Amazonica 5(1): 95-96.

BARROS, R. M., M. M. SAMPAIO, M. F. ASSIS, M. AYRES \& O. R. CUNHA, 1976. General considerations on the karyotypic evolution of Chelonia from the Amazon region of Brazil. Cytologia 41: 559-568.

CUNHA, O. R., 1958. I. Lacertílios da Amazônia. Sobre a ocorrência do gênero Bachia Gray, 1845, na Amazônia brasileira. Boletim do Museu Paraense Emílio Goeldi, nova série Zoologia 11: 1-12.

CUNHA, O. R., 1961. II. Lacertílios da Amazônia. Os lagartos da Amazônia brasileira, com especial referência aos representados na coleção do Museu Goeldi. Boletim do Museu Paraense Emílio Goeldi, nova série Zoologia 39: 1-189.

CUNHA, O. R., 1966. Sobre uma nova espécie de lagarto do Estado de Minas Gerais, Placosoma cipoense sp. n. (Lacertilia: Teiidae). Boletim Museu Paraense Emílio Goeldi, nova série Zoologia 61: 1-9.

CUNHA, O. R., 1967. Lacertílios da Amazônia. III. O gênero "Arthrosaura" Boulenger, 1885. (Lacertilia, Teiidae). In: Atas do Simpósio sobre a Biota Amazónica: 5 (Zoologia): 141-170. CNPq, Rio de Janeiro.

CUNHA, O. R., 1967. Ofídios da Amazônia. I. A ocorrência de Bothrops bilineatus bilineatus (Wied, 1825) nas matas dos arredores da cidade de Belém, Pará. Boletim do Museu Paraense Emílio Goeldi, nova série Zoologia 66: 1-12.

CUNHA, O. R., 1968. Um teratódimo deródimo em gibóia (Constrictor constrictor constrictor) (Linn., 1766) (Ophidia; Boidae). Boletim do Museu Paraense Emílio Goeldi, nova série Zoologia 67: 1-17.

CUNHA, O. R., 1970. Lacertílios da Amazônia. IV. Um novo gênero e espécie de lagarto do Território Federal do Amapá (Lacertilia: Teiidae). Boletim do Museu Paraense Emílio Goeldi, nova série Zoologia 74: 1-8.

CUNHA, O. R., 1970. Uma nova subespécie de quelônio, Kinosternon scorpioides carajasensis da Serra dos Carajás, Pará (Testudinata-Kinosternidae). Boletim do Museu Paraense Emílio Goeldi, nova série Zoologia 73: 1-12.

CUNHA, O. R., 1971. Lacertílios da Amazônia, V. Sobre Gonatodes annularis Boulenger e Neusticurus racenisi Roze, no Brasil. (Lacertilia: Gekkonidae). Revista Brasileira de Biologia 31(1): 113-117.

CUNHA, O. R., 1973. Domingos Soares Ferreira Penna. Uma análise de sua vida e de sua obra. In: O. R. CUNHA (Org.): Obras completas de Domingos Soares Ferreira Penna: 1: 11-41. Conselho Estadual de Cultura, Belém.

CUNHA, O. R., 1974. $108^{\circ}$ Aniversário do Museu Paraense Emílio Goeldi, síntese de sua história. Revista de Cultura do Pará 4(1617): 151-173.
CUNHA, O. R., 1975. Sobre a ocorrência da Tartaruga de couro Dermochelys cariacea (Linnaeus, 1758) na foz do rio Amazonas (Chelonia: Dermochelydae). Boletim do Museu Paraense Emílio Goeldi, nova série Zoologia 81: 1-16.

CUNHA, O. R., 1976. Estampas de Antônio José Landi. In: A. MEIRA FILHO (Ed.): Landi esse desconhecido (O naturalista): 193-202. Conselho Federal de Cultura, Belém.

CUNHA, O. R., 1977. Lacertílios da Amazônia. VI. Uma nova espécie de lagarto Colobosaura landii da região leste do Pará. (Lacertilia: Teiidae). Boletim do Museu Paraense Emílio Goeldi, nova série Zoologia 86: 1-13.

CUNHA, O. R., 1981. VII. Lagartos da região nordeste do Território de Roraima, Brasil. (Lacertilia: Gekkonidae, Scincidae e Teiidae). Boletim do Museu Paraense Emílio Goeldi, nova série Zoologia 107: 1-25.

CUNHA, O. R., 1981. Lacertílios da Amazônia. VIII. Sobre Ophryoessoides tricristatus Duméril, 1851, com redescrição da espécie e notas sobre a ecologia e distribuição na região leste do Pará (Lacertilia: Iguanidae). Boletim do Museu Paraense Emílio Goeldi, nova série Zoologia 108: 1-23.

CUNHA, O. R., 1983. Emílio Augusto Goeldi (1859-1917). Ciência e Cultura 35(12): 1965-1972.

CUNHA, O. R., 1986. Histórico do Museu Paraense Emílio Goeldi. In: G. M. DE LA PENHA, S. A. BRUNI \& N. PAPAVERO (Eds.): O Museu Paraense Emílio Goeldi: 7-19. Banco Safra, São Paulo.

CUNHA, O. R., 1989. Talento e atitude: estudos biográficos do Museu Emílio Goeldi. I: 1-159. MPEG (Coleção Alexandre Rodrigues Ferreira), Belém.

CUNHA, O. R., 1991. Biografias de Domingos Soares F. Penna, Emílio A. Goeldi e E. Snethlage In: WINTERS, Christopher (Ed.): International dictionary of Anthropologists. Garland Pub, New York.

CUNHA, O. R., 1991. O naturalista Alexandre Rodrigues Ferreira. Uma análise comparativa de sua Viagem Filosófica (17831793) pela Amazônia e Mato Grosso com a de outros naturalistas posteriores: 1-88. MPEG (Coleção Alexandre Rodrigues Ferreira), Belém.

CUNHA, O. R., 2009. Jacques Huber (1867-1914). Boletim do Museu Paraense Emílio Goeldi. Ciencias Humanas 4(3): 489-502.

CUNHA, O. R. \& T. X. BASTOS, 1973. A contribuição do Museu Paraense Emílio Goeldi à meteorologia na Amazônia. Publicações Avulsas do Museu Paraense Emílio Goeldi 23: 1-42.

CUNHA, O. R., J. S. LIMA-VERDE \& A. C. M. LIMA, 1991. Novo gênero e espécie de lagarto do Estado do Ceará (Lacertilia: Teiidae). Boletim do Museu Paraense Emílio Goeldi, série Zoologia 7(2): 163-176.

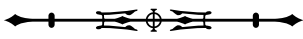


CUNHA, O. R. \& F. P. NASCIMENTO, 1970. Ofídios da Amazônia. II. Liophis miliaris (Linnaeus, 1758) na Amazônia norte oriental (Território Federal do Amapá). (Ophidia: Colubridae). Boletim do Museu Paraense Emílio Goeldi, nova série Zoologia 70: 1-6.

CUNHA, O. R. \& F. P. NASCIMENTO, 1972. Ofidios da Amazônia. III. A ocorrência de Bothrops lichenosus Roze, 1958, no Brasil (Ophidia: Crotalidae). Revista Brasileira de Biologia 32(1): 27.

CUNHA, O. R. \& F. P. NASCIMENTO, 1973. Ofídios da Amazônia. IV. As cobras corais (gênero Micrurus) da região leste do Pará. (Ophidia: Elapidae) Nota preliminar. Publicações Avulsas do Museu Paraense Emílio Goeldi 20: 273-286.

CUNHA, O. R. \& F. P. NASCIMENTO, 1975. Ofídios da Amazônia. V. Bothrops lichenosus Roze, 1958, sinônimo de Bothrops castelnaudi Duméril, Bibron \& Duméril, com nova descrição e comentários. Boletim do Museu Paraense Emílio Goeldi, nova série Zoologia 80: 1-14.

CUNHA, O. R. \& F. P. NASCIMENTO, 1975. Ofídios da Amazônia. VI. Liotyphlops ternetzii (Boulenger, 1896), ofídio raro e de hábitos subterrâneos, na região leste do Pará. (Ophidia: Anomalepididae). Boletim do Museu Paraense Emílio Goeldi, nova série Zoologia 82: $1-8$

CUNHA, O. R. \& F. P. NASCIMENTO, 1975. Ofídios da Amazônia. VII. As serpentes peçonhentas do gênero Bothrops (jararaca) e Lachesis (surucucu) da região leste do Pará. (Ophidia: Viperidae). Boletim do Museu Paraense Emílio Goeldi, nova série Zoologia 83: 1-42.

CUNHA, O. R. \& F. P. NASCIMENTO, 1976. Ofídios da Amazônia. VIII. A ocorrência de Rhinobothryum lentiginosum (Scopoli, 1785) nas proximidades de Belém, Pará. (Ophidia: Colubridae). Boletim do Museu Paraense Emílio Goeldi, nova série Zoologia 84: 1-6.

CUNHA, O. R. \& F. P. NASCIMENTO, 1976. Ofídios da Amazônia. IX. O gênero Liophis Wagler, 1830, na região leste do Pará. (Ophidia: Colubridae). Boletim do Museu Paraense Emílio Goeldi, nova série Zoologia 85: 1-32.

CUNHA, O. R. \& F. P. NASCIMENTO, 1978. Ofídios da Amazônia. $X$. As cobras da região leste do Pará. Publicações Avulsas do Museu Paraense Emílio Goeldi 32: 1-218.

CUNHA, O. R. \& F. P. NASCIMENTO, 1980. Ofídios da Amazônia. XI. Ofídios de Roraima e notas sobre Erythrolamprus bauperthuisii Duméril, Bibron \& Duméril, 1954, sinônimo de Erythrolamprus aesculapii (Linnaeus, 1758). Boletim do Museu Paraense Emílio Goeldi, nova série Zoologia 102: 1-21.

CUNHA, O. R. \& F. P. NASCIMENTO, 1981. Ofídios da Amazônia. XIII. Observações sobre a viviparidade em ofídios do Pará e Maranhão. (Ophidia: Aniliidae, Boidae, Colubridae e Viperidae). Boletim do Museu Paraense Emílio Goeldi, nova série Zoologia 109: 1-20.
CUNHA, O. R. \& F. P. NASCIMENTO, 1982. Ofídios da Amazônia XIV. As espécies de Micrurus, Bothrops, Lachesis e Crotalus do sul do Pará e oeste do Maranhão, incluindo áreas de cerrado desse Estado. (Ophidia: Elapidae e Viperidae). Boletim do Museu Paraense Emílio Goeldi, nova série Zoologia 112: 1-58.

CUNHA, O. R. \& F. P. NASCIMENTO, 1982. Ofídios da Amazônia. XV. As espécies de Chironius da Amazônia oriental (Pará, Amapá e Maranhão). Memórias do Instituto Butantan 46: 139-172

CUNHA, O. R. \& F. P. NASCIMENTO, 1982. Ofídios da Amazônia. XVI. A espécie Uromacerina ricardinii (Peracca, 1897) na Amazônia oriental (leste do Pará). (Ophidia: Colubridae). Boletim do Museu Paraense Emílio Goeldi, nova série Zoologia 113: 1-9.

CUNHA, O. R. \& F. P. NASCIMENTO, 1982. Ofídios da Amazônia. XVII. Revalidação de Micrurus ornatissimus Jan, 1858) diferenciada de M. langsdorffi (Wagler, 1824) e distribuição geográfica das duas espécies (Ophidia: Elapidae). Boletim do Museu Paraense Emílio Goeldi, nova série Zoologia 116: 1-19.

CUNHA, O. R. \& F. P. NASCIMENTO, 1982. Ofídios da Amazônia. XVIII. O gênero Chironius Fitzinger na Amazonia oriental (Ophidia: Colubridae). Boletim do Museu Paraense Emílio Goeldi, nova série Zoologia 119: 1-17.

CUNHA, O. R. \& F. P. NASCIMENTO, 1983. Ofídios da Amazônia. XIX. As espécies de Oxyrhopus Wagler, com uma subespécie nova, e Pseudoboa Schneider na Amazônia oriental e Maranhão. (Ophidia: Colubridae). Boletim do Museu Paraense Emílio Goeldi, nova série Zoologia 122: 1-47.

CUNHA, O. R. \& F. P NASCIMENTO, 1983. Ofídios da Amazônia. XX. As espécies de Atractus Wagler, 1848, na Amazônia oriental e Maranhão (Ophidia: Colubridae). Boletim do Museu Paraense Emílio Goeldi, nova série Zoologia 123: 38.

CUNHA, O. R. \& F. P. NASCIMENTO, 1984. Ofídios da Amazônia. $\mathrm{XXI}$. Atractus zidoki na região leste do Pará e notas sobre $A$. alphonsehogei e A. schach (Ophidia: Colubridae). Boletim do Museu Paraense Emílio Goeldi, série Zoologia 1(2): 219-228.

CUNHA, O. R. \& F. P. NASCIMENTO, 1991. Ofídios da Amazônia. XXII. Revalidação e redescrição de Micrurus albicinctus Amaral, de Rondônia, e sobre a validade de Micrurus waehnerorum Meise, do Amazonas (Ophidia: Elapidae). Boletim do Museu Paraense Emílio Goeldi, série Zoologia 7(1): 43-52.

CUNHA, O. R. \& F. P. NASCIMENTO, 1993. Ofídios da Amazônia. As cobras da região leste do Pará. Boletim do Museu Paraense Emílio Goeldi, série Zoologia 9(1): 1-191.

CUNHA, O. R., F. P. NASCIMENTO \& A. R. HOGE, 1980. Ofídios da Amazônia. XII. Uma subespécie de Sibynomorphus mikani [sic!] do noroeste do Maranhão (Ophidia: Colubridae, Dipsadinae). Boletim do Museu Paraense Emílio Goeldi, nova série Zoologia 103: 1-15.

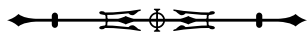


CUNHA, O. R., F. P. NASCIMENTO \& T. C. S. AVILA-PIRES, 1985. Os Répteis da área de Carajás. (Testudines e Squamata). Publicações Avulsas do Museu Paraense Emílio Goeldi, Zoologia 40: 1-100.

CUNHA, O. R. \& F. C. NOVAES, 1981. Área de vertebrados do Museu Paraense Emílio Goeldi, Herpetologia In: O INPA e o Museu Goeldi nos 30 anos do CNPq. Acta Amazonica 2(1): 183-187. Suplemento.

CUNHA, O. R., P. M. TOLEDO \& C. W. MAURITY, 1990. História da pesquisa geológica no Museu Paraense Emílio Goeldi. In: M. M. LOPES \& S. F. M. FIGUEIRÔA (Orgs.): O conhecimento geológico na América Latina: Questões de história e teoria: 115120. Universidade Estadual de Campinas, Campinas.

FERREIRA, C. S. \& O. R. CUNHA, 1957. Contribuição à Paleontologia do Estado do Pará. Notas sobre a Formação Pirabas, com descrição de novos invertebrados fósseis. I - (MolluscaGastropoda). Boletim do Museu Paraense Emílio Goeldi, nova série Geologia 2: 1-61.

FERREIRA, C. S. \& O. R. CUNHA, 1957. Contribuição à Paleontologia do Estado do Pará. Redescrição e novas ocorrências do Dentalium paulini Maury, 1924, na área da Formação Pirabas. II. (Mollusca-Scaphopoda). Boletim do Museu Paraense Emílio Goeldi, nova série Geologia 3: 1-11.

FERREIRA, C. S. \& O. R. CUNHA, 1957. Contribuição à Paleontologia do Estado do Pará. Novos invertebrados fósseis e redescrições de mais duas espécies da Formação Pirabas. III. (Mollusca-Gastropoda). Boletim do Museu Paraense Emílio Goeldi, nova série Geologia 4: 1-33.

FERREIRA, C. S. \& O. R. CUNHA, 1959. Contribuição à Paleontologia do Estado do Pará. Novas contribuições sobre a Formação Pirabas e descrição de novos invertebrados Fósseis. V. (Mollusca-Pelecypoda). Boletim do Museu Paraense Emílio Goeldi, nova série Geologia 8: 1-76.

NASCIMENTO, F. P., T. C. S. AVILA-PIRES \& O. R. CUNHA, 1987. Os Répteis da área de Carajás, Pará, Brasil (Squamata). II. Boletim do Museu Paraense Emílio Goeldi, série Zoologia 3(1): 1-65.

NASCIMENTO, F. P., T. C. S. AVILA-PIRES \& O. R. CUNHA, 1988. Répteis Squamata de Rondônia e Mato Grosso, coletados através do programa POLONOROESTE. Boletim do Museu Paraense Emílio Goeldi, série Zoologia 4(1): 21-66.

SAMPAIO, M. M., R. M. BARROS, M. AYRES \& O. R. CUNHA, 1969. Contribuição cromossômica de quelônios da região Amazônica brasileira. II. Estudos preliminares do gênero Geochelone Fitzinger, 1835. Ciência e Cultura 21(2): 276.

SAMPAIO, M. M., R. M. BARROS, M. AYRES, \& O. R. CUNHA, 1971. A karyological study of two species of turtles from the Amazon region of Brazil. Cytologia 36(2): 199-204.

\section{TRABALHOS EM JORNAIS DE BELÉM OU SIMILARES}

CUNHA, O. R., 1954. Algumas observações sobre o comportamento da pipira em seu habitat. O Estado do Pará, 15 jul.

CUNHA, O. R., 1954. A Biblioteca do Museu Paraense. Um templo da Ciência. O Estado do Pará, 31 out.

CUNHA, O. R., 1954. Biografia da Amazônia. Capítulo I. O Estado do Pará, 17 ago.

CUNHA, O. R., 1954. Biografia da Amazônia. Capítulo II. O Estado do Pará, 22 ago.

CUNHA, O. R., 1954. Biografia da Amazônia. Capítulo III. O Estado do Pará, 29 ago.

CUNHA, O. R., 1954. Biografia da Amazônia. Capítulo IV. O Estado do Pará, 5 set.

CUNHA, O. R., 1954. Biografia de Antônio Corrêa de Lacerda. O Estado do Pará, 19 set.

CUNHA, O. R., 1954. Um Botânico na Amazônia. O Estado do Pará, 7 nov.

CUNHA, O. R., 1954. O cientista solitário. O Estado do Pará, 13 jun.

CUNHA, O. R., 1954. O Círio de Nazaré na opinião de alguns naturalistas. O Estado do Pará, 10 out.

CUNHA, O. R., 1954. O desastre de Von Martius no rio Amazonas. O Estado do Pará, 14 out.

CUNHA, O. R., 1954. O fundador da Zoogeografia do Brasil. A Província do Pará, 25 jul.

CUNHA, O. R., 1954. Os grandes Lacertílios da Amazônia. Capítulo I. O Estado do Pará, 12 dez.

CUNHA, O. R., 1954. Os grandes Lacertílios da Amazônia. Capítulo II. O Estado do Pará, 22 dez.

CUNHA, O. R., 1954. Os grandes Lacertílios da Amazônia. Capítulo III. O Estado do Pará, 28 dez.

CUNHA, O. R., 1954. João Barbosa Rodrigues. Capítulo I. O Estado do Pará, 21 nov.

CUNHA, O. R., 1954. João Barbosa Rodrigues. Capítulo II. O Estado do Pará, 28 nov.

CUNHA, O. R., 1954. João Barbosa Rodrigues. Capítulo III. O Estado do Pará, 5 dez.

CUNHA, O. R., 1954. Leonardo da Vinci como naturalista. O Estado do Pará, 12 set. 
CUNHA, O. R., 1954. Lysenko ou Mendel. O Estado do Pará, 1 ago.

CUNHA, O. R., 1954. Mimetismo em Lepidópteros da Amazônia. O Estado do Pará, 19 nov.

CUNHA, O. R., 1954. Motivos que impediram Humboldt de visitar a Amazônia. O Estado do Pará, 26 set.

CUNHA, O. R., 1954. A origem da medicina tropical. Capítulo I. O Estado do Pará, 19 out.

CUNHA, O. R., 1954. A origem da medicina tropical. Capítulo II. O Estado do Pará, 24 out. c. 2.

CUNHA, O. R., 1954. O primeiro naturalista na Amazônia. O Estado do Pará, 8 ago.

CUNHA, O. R., 1954. Um Rei Naturalista. A Província do Pará, 16 maio.

CUNHA, O. R., 1954. A respiração do peixe-boi. O Estado do Pará, 25 jul.

CUNHA, O. R., 1955. I. Os grandes Lacertílios da Amazônia. Capítulo IV. O Estado do Pará, 24 fev.

CUNHA, O. R., 1955. I. Os grandes Lacertílios da Amazônia. Capítulo V. O Estado do Pará, 13 mar.

CUNHA, O. R., 1955. I. Os grandes Lacertílios da Amazônia. Capítulo VI. O Estado do Pará, 23 mar.

CUNHA, O. R., 1955. I. Os grandes Lacertílios da Amazônia. Capítulo VII. O Estado do Pará, 8 abr.

CUNHA, O. R., 1955. II. Lacertílios da Amazônia. A Palavra, 1 jan.

CUNHA, O. R., 1955. II. Lacertílios da Amazônia. A Palavra, 27 fev.

CUNHA, O. R., 1955. III. Lacertílios da Amazônia. Sobre a oviviparidade do lagarto-cobra (Mabuya mabouya mabouya (Lacépede), 1788. A Palavra, 29 maio.

CUNHA, O. R., 1955. IV. Lacertílios da Amazônia. O Estado do Pará, 17 mar.

CUNHA, O. R., 1955. V. Lacertílios da Amazônia. O Estado do Pará, 29 mar.

CUNHA, O. R., 1955. VI. Lacertílios da Amazônia. O Estado do Pará, 15 abr.

CUNHA, O. R., 1959. O Museu Paraense e a Geologia do Pará I. A Província do Pará, 28 ago.

CUNHA, O. R., 1959. O Museu Paraense e a Geologia do Pará II. A Província do Pará, 30 ago.
CUNHA, O. R., 1959. O Museu Paraense e a Geologia do Pará III (Conclusão). A Província do Pará, 1 set.

CUNHA, O. R., 1960. Alexandre Von Humboldt e a Amazônia. A Província do Pará, 6 mar.

CUNHA, O. R., 1960. Antônio Corrêa de Lacerda. A Província do Pará, 31 jan.

CUNHA, O. R., 1961. Esboço biográfico de um cientista - Walter Egler. A Província do Pará, 17 set.

CUNHA, O. R., 1965. O Centenário do Museu Paraense I. As origens. A Província do Pará, 17 out.

CUNHA, O. R., 1965. O Centenário do Museu Paraense "Emílio Goeldi" II. Os fundamentos e evolução histórica. A Província do Pará, 20 out.

CUNHA, O. R., 1965. O centenário do Museu Paraense "Emílio Goeldi" III. Ainda o museu de 1866 a 1871. A Província do Pará, 24 out.

CUNHA, O. R., 1965. O centenário do Museu Paraense "Emílio Goeldi" IV. O Museu de 1871 a 1891. A Província do Pará, 31 out.

CUNHA, O. R., 1965. O centenário do Museu Paraense "Emílio Goeldi" V. Ainda o Museu de 1871 a 1881 [1891]. A Província do Pará, 7 nov.

CUNHA, O. R., 1965. O centenário do Museu Paraense "Emílio Goeldi” VI. O Museu de 1871 a 1891 (continuação). A Província do Pará, 14 nov.

CUNHA, O. R., 1965. O centenário do Museu Paraense "Emílio Goeldi” VIII [VII]. O Museu de 1871 a 1891 (continuação). A Província do Pará, 21 nov.

CUNHA, O. R., 1965. O centenário do Museu Paraense "Emílio Goeldi” VIII. O Museu de 1871 a 1891. A Província do Pará, 28 nov.

CUNHA, O. R., 1965. O centenário do Museu Paraense "Emílio Goeldi” IX. O Museu de 1871 a 1891 (continuação). A Província do Pará, 5 dez.

CUNHA, O. R., 1965. O centenário do Museu Paraense "Emílio Goeldi”. A Província do Pará, 12 dez.

CUNHA, O. R., 1966. Museu Paraense Emílio Goeldi: Um século de História e Ciência I. A Província do Pará, 6 out.

CUNHA, O. R., 1966. Museu Paraense Emílio Goeldi: Um século de História e Ciência II. A Província do Pará, 16 out.

CUNHA, O. R., 1966. Museu Paraense Emílio Goeldi: Um século de História e Ciência 3. A Província do Pará, 17 out.

CUNHA, O. R., 1966. Museu Paraense Emílio Goeldi: Um século de História e Ciência 4. A Província do Pará, 6 nov. 
CUNHA, O. R., 1967. Augusto Montenegro e o Museu Paraense. A Província do Pará, 18 jul.

CUNHA, O. R., 1967. Centenário da abertura do rio Amazonas (1867-1967). A Província do Pará, 21 maio.

CUNHA, O. R., 1969. O Museu Paraense Emílio Goeldi e a Atualidade da Amazônica. A Província do Pará, 28 e 29 set.

CUNHA, O. R., 1972. O Museu e a febre amarela. O Liberal: 2, 11 ago.

CUNHA, O. R., 1974. 108 aniversário do Museu Paraense Emílio Goeldi. A Província do Pará, 13 out.

CUNHA, O. R., 1974. $108^{\circ}$ aniversário do Museu Paraense Emílio Goeldi. Correio Braziliense, Brasília, 18 out.

CUNHA, O. R., 1974. $108^{\circ}$ aniversário do Museu Paraense Emílio Goeldi. O Diário do Congresso Nacional, Brasília, out.

CUNHA, O. R., 1976. A criação do Pirarucu nos lagos do Museu. O Liberal, 24 maio.

CUNHA, O. R., 1978. Pesquisas herpetológicas no Museu Paraense Emílio Goeldi. A Província do Pará, 16 abr. Caderno 2: 6.

CUNHA, O. R., 1984. Viagem Filosófica pelas capitanias do GrãoPará, Rio Negro, Mato Grosso e Cuiabá (1783/1793). Bicentenário da chegada a Belém de Alexandre Rodrigues Ferreira. O Liberal, 15 nov.

CUNHA, O. R., 1985. Maria Emília Snethlage (1868-1929): a primeira mulher cientista na Amazônia. O Liberal, 15 nov.

CUNHA, O. R., 1986. Domingos Soares Ferreira Penna (1818-1888). O Liberal, 29 set.

CUNHA, O. R., 1986. O edifício central do Museu (Pavilhão Domingos S. Ferreira Penna, 1879). O Liberal: 42, 22 set.

CUNHA, O. R., 1986. Emílio Augusto Goeldi (1859-1917). O Liberal, 29 nov.

CUNHA, O. R., 1988. Centenário da morte de Domingos S. Ferreira Penna. O Liberal, 10 jan.
CUNHA, O. R., 1988. O criador do Museu Paraense Emílio Goeldi. O Liberal, 22 jan.

CUNHA, O. R., 1988. O Museu Paraense Emílio Goeldi. O Diploma outorgado pelo Museu Paraense Emílio Goeldi, Histórico. O Diário do Pará, 5 maio. Caderno de cultura: B6-B7.

CUNHA, O. R., 1988. Dr. Jacques Huber (1867-1914): o grande diretor do Museu Paraense Emílio Goeldi. Capítulo I. O Diário do Pará, 28 jul. Caderno de cultura.

CUNHA, O. R., 1988. Dr. Jacques Huber (1867-1914): o grande diretor do Museu Paraense Emílio Goeldi. Capítulo II. O Diário do Pará, Belém, 4 ago. Caderno de cultura.

CUNHA, O. R. \& A. MEIRA FILHO, 1970. Landi, esse desconhecido. Capítulo I. A Província do Pará, 3 dez.

CUNHA, O. R. \& A. MEIRA FILHO, 1970. Landi, esse desconhecido. Capítulo II. A Província do Pará, 20 out.

CUNHA, O. R. \& A. MEIRA FILHO, 1970. Landi, esse desconhecido. Capítulo III. A Província do Pará, 20 out.

CUNHA, O. R. \&A. MEIRA FILHO, 1970. Landi, esse desconhecido. Capítulo IV. A Província do Pará, 3 jan.

CUNHA, O. R. \& A. MEIRA FILHO, 1971. Landi, esse desconhecido. Capítulo V. A Província do Pará, 28 mar.

\section{OUTRAS PUBLICAÇÕES}

CUNHA, O. R., 2009. Apresentação. In: M. I. F. RAMOS, H. M. M. SANTOS, S. A. R. F. COSTA \& P. M. TOLEDO (Eds.): Catálogo de Fósseis: Coleção Paleontológica do Museu Paraense Emílio Goeldi. Museu Paraense Emílio Goeldi, Belém.

\section{AGRADECIMENTOS}

Os autores agradecem a Ligia Maria da Cunha Barbosa pelo acesso aos desenhos e às fotografias de Osvaldo Rodrigues da Cunha, bem como pela autorização para publicá-los. 